\title{
El pretérito imperfecto de subjuntivo en la Península Ibérica del siglo xx
}

\section{The past subjunctive in the Iberian Peninsula of the 20th century}

\author{
Víctor Lara Bermejo \\ Universidad Nacional de Educación a Distancia
}

Resumen. El pretérito imperfecto de subjuntivo ha acaparado numerosos estudios en español debido a la inserción de la forma indicativa $-r a$ en el modo subjuntivo como sinónimo de -se. La mayoría de estos trabajos hacen un estudio diacrónico, centrándose en estadios de la lengua anteriores al siglo XIX. En este artículo, pretendemos dar cuenta de la casuística dialectal del pretérito imperfecto de subjuntivo de mediados del siglo xx, desde un punto de vista iberromance. El análisis de los datos del Atlas lingüístico de la Península Ibérica y su comparación con atlas posteriores y corpus sociolingüísticos arrojará un poco más de luz sobre la realidad de dicho tiempo hace 100 años, ya que se advierte todavía un uso muy mayoritario de -se en el este peninsular, una sistematicidad en $-r a$ en el español occidental y la introducción de $-r a$ por $-s e$ en lenguas en las que no se había atestiguado aún dicha evolución.

Palabras clave: Pretérito imperfecto de subjuntivo; geografía dialectal; iberorromance; ALPI

\begin{abstract}
There are many studies dedicated to the past subjunctive in Spanish due to the insertion of the indicative $-r a$ form into the subjunctive, as a synonym of the $-s e$ form. Most of these studies focus on its diachrony, mainly in language stages prior to the $19^{\text {th }}$ century. In this paper, I will discuss the dialect variation of the past subjunctive in the middle $20^{\text {th }}$ century from an IberoRomance perspective. The analysis of the data from the Atlas lingüístico de la Península Ibérica as well as its comparison to later atlases and corpora will shed more light on this reality 100 years
\end{abstract}

Data de recepción: 08-04-2018 - Data de aceptación: 12-07-2018. 
ago, since it is still possible to observe a considerably higher frequency of $-s e$ in the east of the Iberian Peninsula, a systematic use of $-r a$ in western Peninsular Spanish, and the emergence of $-r a$ instead of $-s e$ in languages where this evolution had not been yet attested.

Keywords: Past subjunctive; dialect geography; Ibero-Romance; ALPI

\section{INTRODUCCIÓN}

La expresión de un tiempo pretérito en modo subjuntivo ha sido compleja en toda la historia del español. En primer lugar, la forma etimológica (-se) ha ido cediendo terreno gradualmente a una nueva que proviene del pluscuamperfecto de indicativo $(-r a)$; en segundo lugar, dialectalmente se documentan distintas soluciones que no encajan con el estándar en la mayoría de los casos: el condicional o el imperfecto de indicativo. Además, en América su empleo es reducido y ha sido prácticamente desplazado por el presente de subjuntivo en ciertos casos de consecutio temporum, en concreto, aquellos en los que el verbo de la principal se flexiona en pasado.

Veiga (2006) hace un recorrido histórico de las formas de subjuntivo del latín al español actual, centrándose en la inserción de $-r a$ en contextos de -se y, en contra de una de las teorías más asentadas, que establece la simetría en los dos elementos de las oraciones condicionales, el autor arguye que la entrada de - ra en -se no es más que otro caso de la irrupción de formas de indicativo a contextos subjuntivos. La teoría de la simetría, no obstante, defendida por Harris (1971), Montero (1989), Narbona (1990), Rojo (1974) y Wright (1933) entre otros, relaciona la introducción de $-r a$ en las prótasis de las condicionales como tendencia universal a mantener el mismo tiempo verbal tanto ahí como en las apódosis. Así, la aparición de -ra en las apódosis produjo su coaparición en las prótasis, lo cual, con el paso del tiempo, derivó en su reinterpretación como sinónimo de las formas en -se, hasta asentar su empleo como lo conocemos hoy día.

Para Veiga (2006), en cambio, la razón de la inserción de $-r a$ a contextos de -se se debe a los dos cambios fundamentales que el español sufrió en su paradigma temporal. Para empezar, es pertinente distinguir entre los tipos de indicativo y subjuntivo. En el primer caso, tenemos el indicativo objetivo, no irreal y no incierto (IND 0) (1-2), el objetivo, no irreal e incierto (IND 1) (3-4), y el objetivo irreal (IND 2) (5 - 6). En el caso del subjuntivo, este se escinde en subjetivo no irreal (SUBJ 0) (7-8), y subjetivo irreal (SUBJ 2) $(9-10)$. 
(1) Ahora canto.

(2) Ayer canté.

(3) Mañana cantaré.

(4) Mañana iría con ellos de buena gana.

(5) Con el buen día que hace hoy, mi prima estaría encantada en la playa.

(6) Con el buen día que hace hoy, mi prima estaba encantada en la playa.

(7) Aunque seas mi hijo, no te lo perdono.

(8) No me gustó que estuviera tan antipático.

(9) Si tuviese dinero, me compraría una casa.

(10) Si hubiera estudiado, habría aprobado.

Observamos que la diferencia esencial en el caso del condicional para el IND 2 radica en el hecho de que dicho tiempo verbal puede conmutarse por un imperfecto de indicativo e incluso una forma en $-r a$. Será el SUBJ 0 el homólogo en dicho modo de los IND 0 y 1 , mientras que el SUBJ 2 es el equivalente en dicho modo del IND 2.

El inicio de -ra como sinónimo de -se data de alrededor del siglo XIII (Ridruejo 1979, Veiga 2006, Cano 1990) y está muy constreñido a oraciones condicionales, lo cual llevó a la teoría de la simetría a la que nos hemos referido. Para Veiga (2006), sin embargo, si las condicionales favorecieran la simetría, esta tendría que darse en cualquier tiempo verbal y no solo en el pretérito, cosa que no pasó. Para el autor, el contexto IND 2 del que partía la forma latina en $-r a$ fue fundamental para colarse en prótasis condicionales donde la oposición objetivo - subjetivo o real - irreal no era relevante. Una vez se asienta en dicho contexto y coocurre con -se, la forma de indicativo empieza a reanalizarse como forma propia del subjuntivo y de pretérito imperfecto. Es entonces cuando salta a valores del SUBJ 0, situación que parece haber tenido lugar alrededor del siglo XVII. A partir de dicha centuria, $-r a$ alcanza valores de subjuntivo no irreales y se vuelve completamente alomórfica de -se, hasta el punto de que -se extiende su valor a lecturas donde previamente no podía aparecer, como en (11).

(11) De buena gana le hubiese dicho lo que pienso de sus estúpidos amigos.

El ejemplo (11) muestra que la forma en -se bien puede conmutarse por - $r a$ o el condicional, si bien este empleo es relativamente moderno, así como la reutilización retórica de - ra para un pluscuamperfecto.

Veiga (2006) insiste en que la implantación de - ra como subjuntivo no es un caso aislado, sino una tendencia cuanto menos panrománica, como el caso del francés o el propio español, que ha sustituido los futuros de subjuntivo por presentes de indicativo en la mayoría de los casos, por citar un ejemplo. En lo que sí hace hincapié es en el 
estadio más conservador del español latinoamericano con la forma en - $r a$, ya que sigue apareciendo en contextos de apódosis de condicionales.

El desplazamiento de $-r a$ de IND 2 a subjuntivo también se atestigua en gallego (Veiga 2006) y en el catalán hablado en zona valenciana (Badia i Margarit 1994, Wheeler et al. 1999, Solà 2002), pero no en otras lenguas romances peninsulares. Sin embargo, carecemos de estudios geolingüísticos que den cuenta efectivamente de la distribución espacial del imperfecto de subjuntivo en distintos contextos sintácticos en los que puede aparecer, ya que resulta de especial interés si una forma predomina sobre la otra o incluso si $-r a$ ha saltado a otras variedades. Para dar cuenta precisamente de la casuística geográfica, pasamos a explicar el corpus y la metodología que hemos empleado a tal efecto.

\section{CORPUS Y METODOLOGÍA}

La primera herramienta dialectal que utilizaremos para cartografiar la situación del imperfecto de subjuntivo es el Atlas lingüistico de la Península Ibérica (ALPI). Dicho trabajo data de mediados del siglo xx y pretendía dar cuenta de la variación léxica, morfosintáctica y fonética de las lenguas romances en todo el territorio peninsular. Este atlas dialectal fue creado por Tomás Navarro Tomás y llevado a cabo por tres equipos repartidos por la zona castellana, la gallego-portuguesa y la catalano-valenciana, con dos investigadores nativos en cada grupo. Se encuestaron 527 enclaves rurales y en cada uno de ellos se entrevistó a un informante con poco nivel de estudio, que rondara entre los cuarenta y sesenta años y que toda su vida hubiera vivido en el pueblo. Las entrevistas fueron realizadas entre los años 1920 y 1950 y consistían en oraciones y palabras preconcebidas que los informantes repetían en su variedad vernácula y cuyos resultados eran manuscritos fonéticamente. La metodología puede plantear dudas acerca de la espontaneidad de las respuestas del informante, así como de los escasos datos sobre los fenómenos vernáculos, ya que tan solo se recogía una respuesta por pregunta y se encuestaba a un solo informante por enclave.

Entre estas oraciones, hay seis preguntas donde se incluye dicho tiempo verbal, a saber: 354. Me pidieron que les ayudase; 386. Si tuviera dinero lo compraria; 387. Si estudiase, aprendería; 388. Si pudiera, la mataría; 389. Yo quisiera ser rico; 390. Ojalá lloviese.

En segundo lugar, sacaremos los datos que hay sobre preguntas que contienen en su formulación un pretérito imperfecto de subjuntivo en los atlas lingüísticos realizados en la segunda mitad del siglo pasado. En concreto, observaremos los datos del ALEC-MAN, ALECANT, ALEA, ALCyL y ALEANR. La metodología de dichos 
atlas imita la del ALPI, pero la recolección de sus datos data de 1970 hasta 1990, por lo que su comparación con los resultados del ALPI arrojará información sobre la evolución del imperfecto de subjuntivo a nivel dialectal a lo largo del siglo xx. Por último, recogeremos datos de corpus sociolingüísticos del portugués, catalán y español. En concreto, analizaremos las ocurrencias que existen en el CORDIAL-SIN, COD y COSER, cuya metodología es común, ya que se basa en entrevistas semidirigidas en las que hablantes mayores, rurales y sin nivel educativo hablan espontáneamente de temas diversos de su vida cotidiana o del día a día en el mundo rural. Los tres corpus datan de finales del pasado siglo y principios de este, por lo que observaremos toda una evolución durante cien años del imperfecto de subjuntivo mediante el cotejo de las herramientas expuestas.

\section{RESULTADOS}

Empecemos describiendo la geolingüística que ofrece el ALPI en sus distintas preguntas. En primer lugar, analizaremos aquellas oraciones donde se explicita en su formulación un imperfecto de subjuntivo.

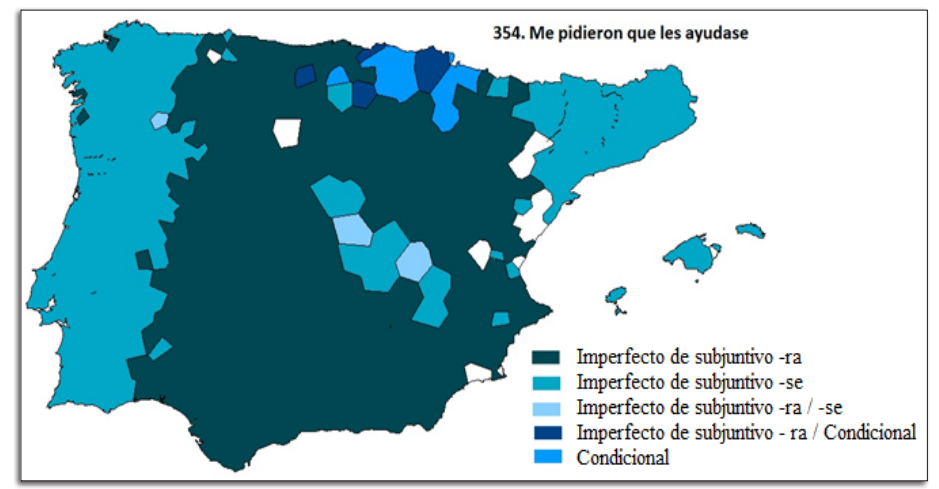

Mapa 1

En el mapa 1, se observa que el verbo de la subordinada puede construirse de tres maneras distintas: -ra, -se y -ría. Es decir, los hablantes han optado por ayudara, ayudase o ayudaría. Solo ciertos enclaves del norte peninsular alternan entre la opción de subjuntivo y de condicional. Sin embargo, la opción casi hegemónica de toda la zona castellana es el uso de $-r a$ como desinencia de imperfecto de subjuntivo, en detrimento de $-s e$, el cual se localiza ininterrumpidamente por las áreas catalana, portuguesa y gallega (salvo esporádicas respuestas de -ra). Es, además, la Comunidad 
Valenciana el único lugar catalanoparlante donde se ha optado por -ra como marca de imperfecto de subjuntivo.

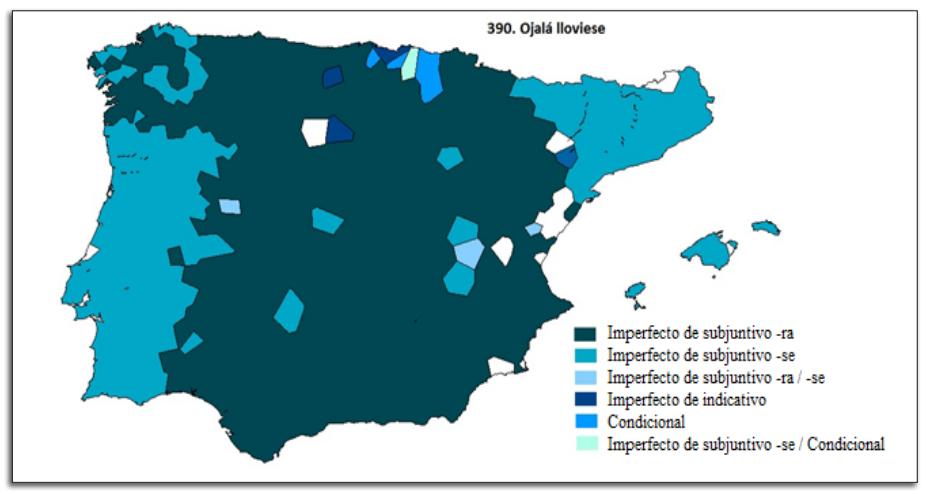

Mapa 2

En el mapa 2, vemos que la distribución de desinencias coincide en gran medida con el mapa 1, salvo la elección del condicional que decrece exponencialmente y se documenta aisladamente en el norte. Igualmente, la alternativa en -se es la única del portugués y el catalán de Cataluña y Baleares, pero es - $r a$ la morfología que se impone sin lugar a dudas en castellano, en valenciano y en gran parte del gallego.

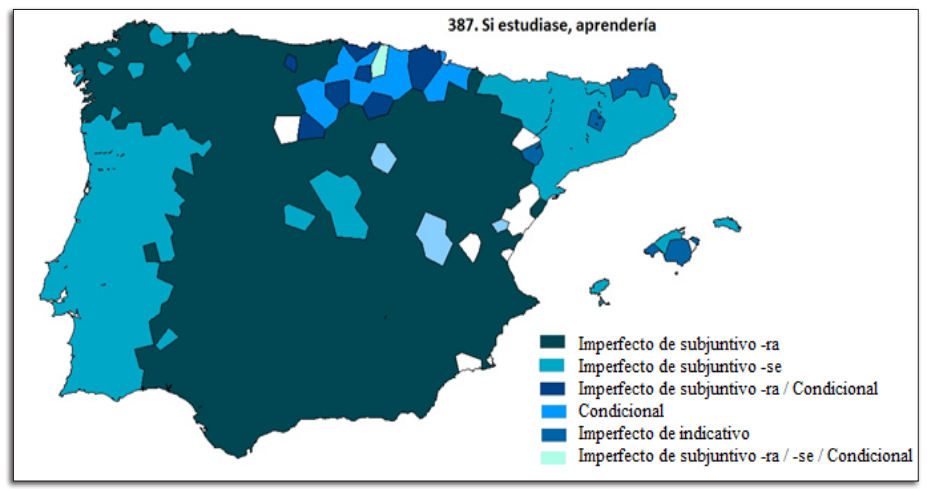

Mapa 3

En el mapa 3, tenemos un imperfecto de subjuntivo ubicado en una subordinada condicional y, a pesar de que la misma formulación de la pregunta prevé el uso de $-s e$, los patrones expuestos en los mapas 1 y 2 se repiten con escasas diferencias. El uso de -se aumenta levemente (siempre en el centro oriental del castellano), -ra se 
impone en Galicia, el condicional crece levemente y es en el catalán de Mallorca y el Rosellón donde se ha preferido un imperfecto de indicativo en lugar de una forma de subjuntivo.

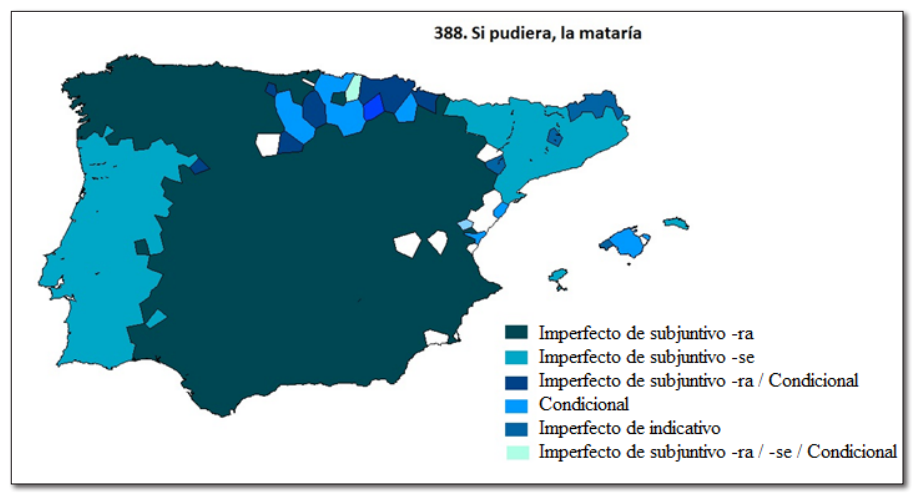

Mapa 4

En el mapa 4, de nuevo el comportamiento es coherente con el de los otros mapas. Y, aunque la pregunta esta vez establezca la desinencia - ra en su enunciado, -se aparece de manera esporádica en castellano en la misma región, si bien es la única opción en Portugal y casi toda Cataluña. Una vez más, la opción del condicional por imperfecto se documenta en el norte peninsular, aunque con mayor fuerza que en los mapas anteriores y, al igual que en el anterior, parte del catalán de Baleares y de Francia opta por un imperfecto de indicativo en lugar del de subjuntivo.

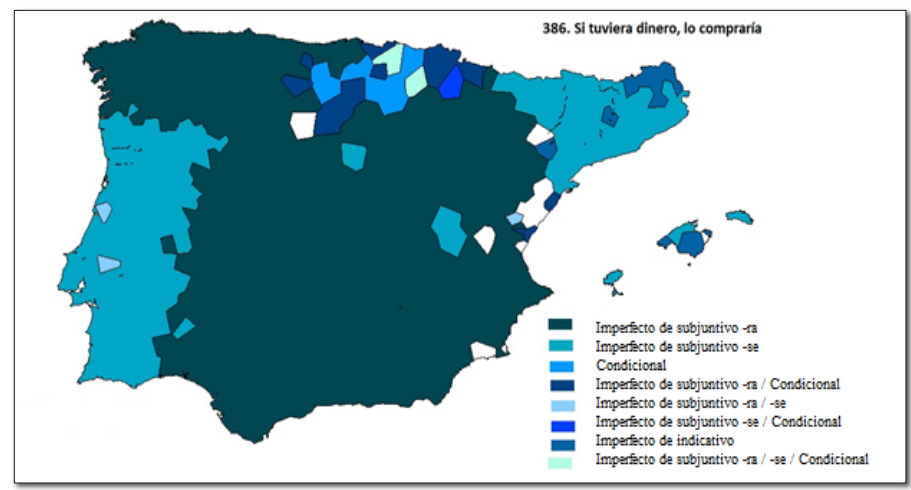

Mapa 5 
El mapa 5 corrobora los patrones mencionados en los anteriores, con el imperfecto de indicativo en el catalán septentrional y balear, el condicional en el norte peninsular, la preferencia por -se en el catalán y el portugués, y la fuerza de $-r a$ en castellano y valenciano. Esta vez, no obstante, se han podido hallar dos enclaves en Portugal que alternan entre $-r a \mathrm{y}-s e$.

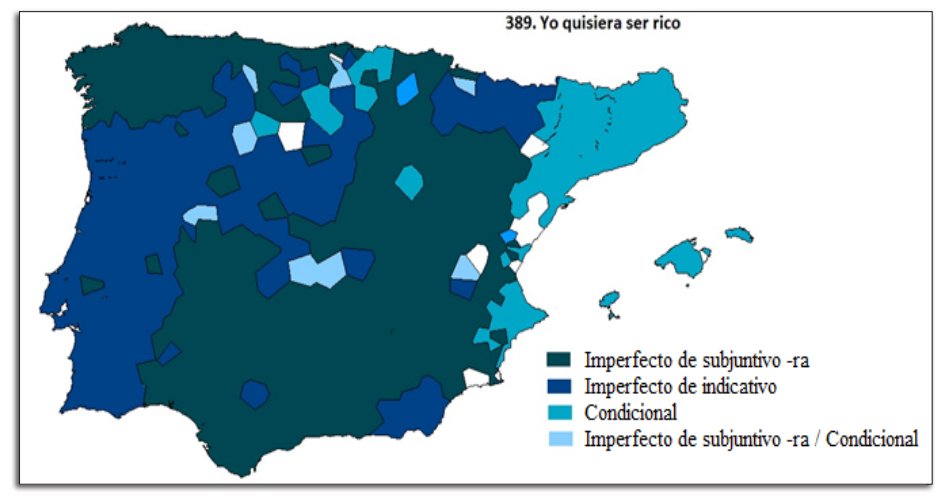

Mapa 6

El mapa 6 muestra un modelo distinto al de los mapas mostrados hasta ahora. En primer lugar, se trata de una oración desiderativa cuyo lexema modal se encuentra en la principal y no en la subordinada. El verbo modal es el único caso en el que se permite un imperfecto de subjuntivo en dicho contexto sintáctico y la misma formulación de la frase incluye dicho tiempo verbal a expensas de un condicional. La ilustración, en este caso, exhibe una gran parte del español que opta por -ra, a lo cual se le suma Galicia y muy anecdóticamente Portugal. En segundo lugar, la opción del imperfecto de indicativo se difunde por el portugués y penetra en el español de Castilla y León, el norte de Aragón y Navarra y el sureste de Andalucía. La opción del catalán, da igual la zona de este, opta por el condicional, el cual también se da, aunque alternando con el imperfecto de subjuntivo, en zonas del norte peninsular.

Pasemos ahora a describir la estrategia de las apódosis de las mismas oraciones condicionales del ALPI. 


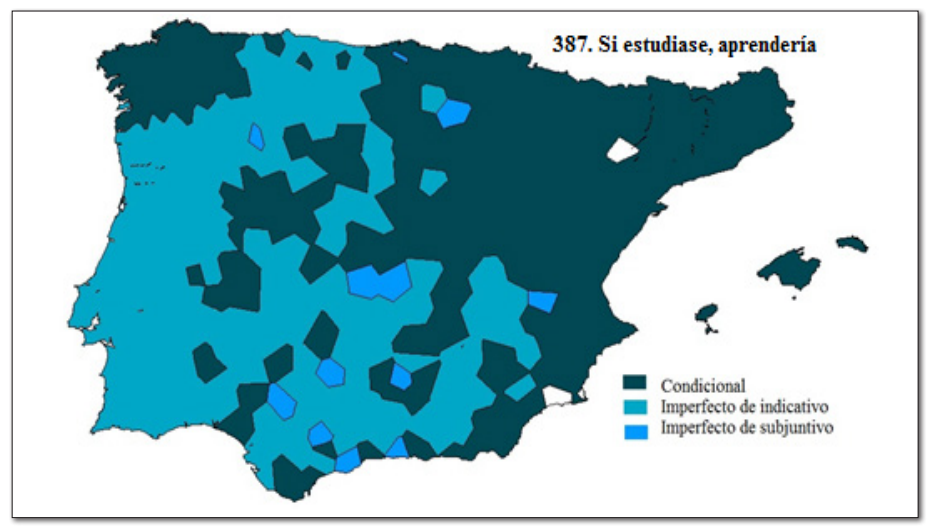

Mapa 7

El mapa 7 indica que el tiempo verbal elegido en la apódosis de la pregunta se reparte entre el condicional (muy asentado en catalán, gallego y el este peninsular) y el imperfecto de indicativo (unánime en Portugal y muy prolífico en el oeste y centro-sur peninsulares). De manera anecdótica surge el imperfecto de subjuntivo (aprendiera) como sinónimo de IND 2, tal y como era la lectura inicial de dicha forma.

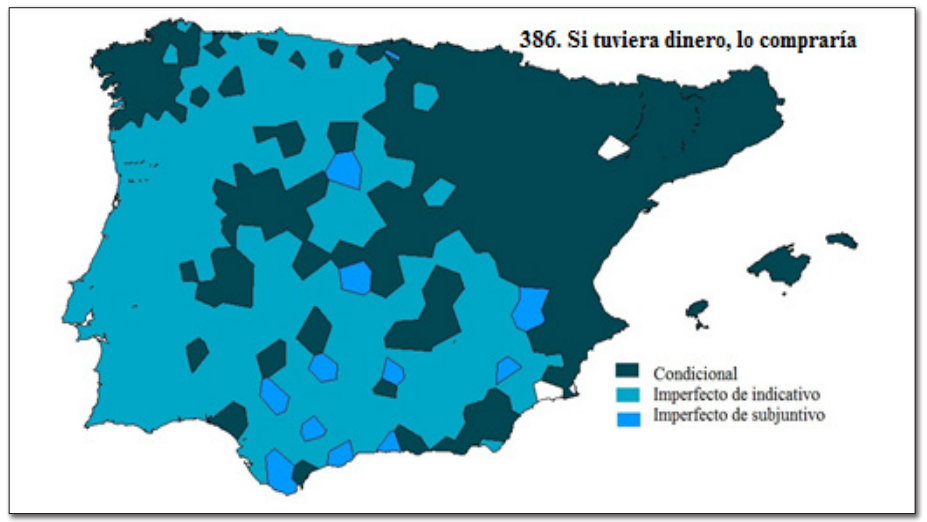

Mapa 8

En este mapa 8, el patrón se repite, ya que el condicional se asienta sobre el catalán y el este peninsular y en Galicia (aunque con una menor incidencia). En su lugar, el imperfecto de indicativo se impone en el oeste y el portugués, mientras que el imperfecto de subjuntivo para IND 2 aparece aisladamente. 


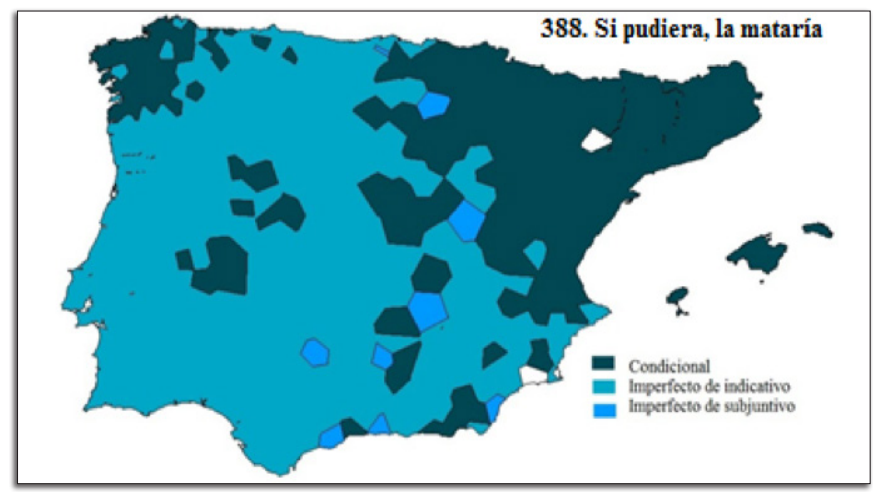

Mapa 9

En el mapa 9, no vemos grandes cambios, ya que el condicional se mantiene férreo en el catalán, el gallego y el español oriental y el imperfecto de indicativo se refuerza en el oeste y el centro peninsulares, el portugués y penetra algo más en el este y sureste, llegando incluso a la zona valenciana. Encontramos esta vez menos ocurrencias de imperfecto de subjuntivo para IND 2.

Una vez descritos los datos del ALPI, pasaremos a exponer los que se han encontrado en los atlas a los que hicimos mención en el apartado de corpus y metodología. Empecemos por el ALEANR. Este atlas posee tres mapas que ofrecen un imperfecto de subjuntivo, a saber: 1704. Si tuviera dinero, lo comprarí; 1705 . Le dije que trajera pan; 1706. Ojalá lloviera.

El primero ofrece un contexto de SUB 2 e IND 2. Los resultados apuntan a un uso productivo del condicional en Navarra y La Rioja, pero el empleo de la forma en -ra en todo Aragón, con excepción de la Franja oscense, que opta por -se. La oración principal, por su parte, siempre se construye con el condicional, excepto en un par de casos aislados, que elige el imperfecto de indicativo. La segunda pregunta, que contiene una subordinada completiva, da cuenta más o menos la misma casuística: condicional en Navarra y Rioja, hegemonía de - ra en Aragón y uso de -se en la Franja oscense aunque penetra en un área mayor de Huesca, y de norte a sur en el centro de Teruel. Por último, la tercera pregunta hace decrecer el condicional en La Rioja, ya que aparece en el noreste (aunque es sistemático en Navarra) y establece - ra para el resto de la geografía, salvo en la mitad oriental de Huesca, donde se prefiere -se, si bien hay que admitir que -se se reparte de manera esporádica por todo Aragón.

Si nos detenemos en el ALECANT, observamos cuatro preguntas: 1216. Si tuviera dinero, lo compraría; 1217. Aunque pudiera, no lo haría; 1218. Le dije que trajera pan; 1220. Ojalá lloviese. 
En la primera pregunta, nos enfrentamos ante un SUBJ 2 y un IND 2. En el primer caso, observamos que la solución en - $r a$ ocupa toda la geografía occidental, mientras que las zonas centrales, orientales y meridionales prefieren el condicional. No se ven ocurrencias de -se cuando la alternativa recae en el imperfecto de subjuntivo. En el segundo caso, no obstante, las soluciones de imperfecto de indicativo y de condicional alternan en casi toda la región, con una intensidad levemente menor en el condicional. La segunda pregunta, cuyos tiempos verbales coinciden en la formulación con los modos de la primera, exhibe un patrón distinto. No nos hallamos ante una oración condicional, sino concesiva y esto ha provocado el descenso del condicional en la subordinada, ya que solo el este de Cantabria ha optado por podría frente a pudiera. Lo mismo ocurre con la principal, cuya alternativa en imperfecto de indicativo ha decrecido y solo surge en el centro y el suroeste de la comunidad autónoma. En contraposición, es el condicional el que se impone.

La pregunta que incluye el imperfecto de subjuntivo en una subordinada completiva se comporta como un SUBJ 2. En esta ocasión, -ra se impone (no hay rastro de $-s e$ ), pero el condicional sigue surgiendo en el este y sureste de la región. Por último, la oración desiderativa exhibe tan solo $-r a$, aunque con ocurrencias esporádicas de - se. En este caso, es el condicional el que no aparece.

El ALEA tan solo da cuenta de una pregunta: 1853. Si tuviera dinero, compraría un coche, mientras que el ALEC-MAN dispone de cuatro preguntas: SIN-43. Si tuviera dinero, lo compraría; SIN-45. Ojalá lloviese; SIN-103. Eso me lo comería ahora mismo; SIN-104. Si lo hubiera sabido, habría venido. El mapa del ALEA permite observar el uso de SUBJ 2 e IND 2 (mapa 9). Existe unanimidad de - ra en toda la región, sin ningún registro de $-s e$, cuando el informante ha optado por la forma de subjuntivo.

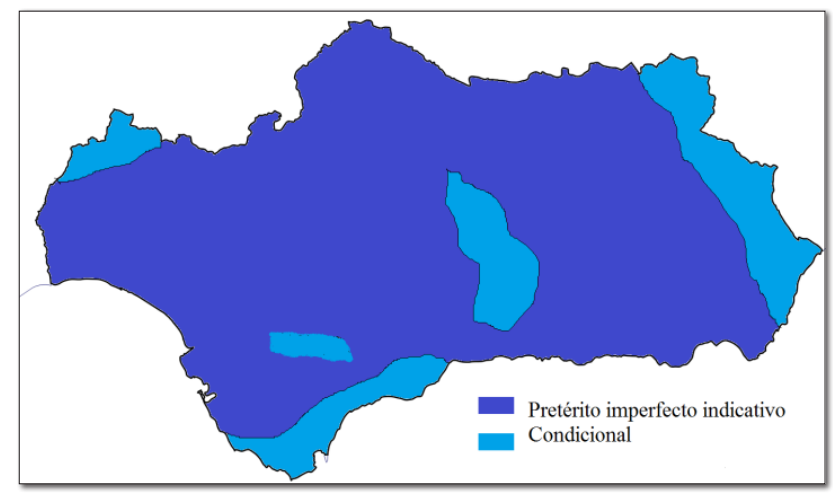

Mapa 9 
La discrepancia se refleja en el IND 2, ya que el uso del imperfecto de indicativo está bastante asentado, si bien el condicional o convive o desplaza a este en zonas del centro andaluz, la Costa del Sol, la sierra de Ronda, el noroeste de Huelva y puntos de Andalucía oriental en contacto con otras regiones.

Para el ALEC-MAN, no obstante, tenemos otros datos y es el único que ha previsto un SUBJ 2 con un tiempo compuesto. En la primera pregunta, el uso de $-r a$ es hegemónico, con escasas ocurrencias de $-s e$. La forma de IND 2 ha sido el condicional. En el caso de la oración desiderativa, se advierte un patrón idéntico, es decir, una amplia mayoría de respuestas en - ra y una ínfima en -se sin ningún modelo geográfico definido. La pregunta con un condicional, es decir, con un IND 2, ofrece mayor variedad. Se observa una convivencia casi total de condicional e imperfecto de indicativo en toda el área encuestada, con esporádicas respuestas unívocas ya sea a favor del imperfecto como del condicional, sin que se perciba necesariamente un mayor uso de una u otra alternativa en función de la ubicación. Por último, la pregunta SIN-104 da cuenta del tiempo verbal elegido en la principal. De acuerdo con el mapa resultante, las soluciones con el condicional son minoritarias y no se concentran en ningún punto concreto de la geografía castellano-manchega. En su lugar se reparten el pretérito pluscuamperfecto de subjuntivo (hubiera venido), con algunas ocurrencias alomórficas en -se y el condicional compuesto (habría venido). De nuevo, estas dos soluciones se reparten casi a partes iguales, pero sin seguir ningún patrón cartográfico específico.

Para el Atlas Lingüistico de Castilla y León, encontramos preguntas muy similares: 148. Si hubiese tenido dinero, lo compraría; 149. Aunque pudiera, no lo haría; 150. Le dijo que trajera un pan; 151. Esto te lo dije para que fueras bueno. Los mapas de dicho atlas muestran una coherencia en el uso del condicional en lugar del imperfecto de subjuntivo en las provincias de Burgos, Soria, Palencia y, esporádicamente, en el norte de Segovia. Asimismo, los enclaves que alternan con el imperfecto o donde existe este tiempo en las preguntas formuladas presentan el alomorfo $-r a$ $\mathrm{y}$, en escasas ocasiones, $-\mathrm{se}$. La alternativa del tiempo compuesto presenta el mismo comportamiento que en otros atlas, ya que la solución hegemónica es con -ra y, de manera minoritaria, con -se o con el condicional.

Si nos fijamos en la alternancia alomórfica entre - ra y -se en el COSER, advertimos que todas las provincias encuestadas cuentan con una respuesta hegemónica de $-r a$ y, en muy pocos casos, de -se. Es más, las producciones espontáneas de los informantes procedentes de la zona oriental no favorecen en absoluto el alomorfo -se, sino que - $r a$ se mantiene férreamente, hasta tal punto que hallamos enclaves en los que no se explicita nunca una forma en $-s e$. Las ocurrencias disponibles de provincias 
bilingües tampoco han mostrado una preferencia por -se; todo lo contrario, -ra es tan vigente como en plena zona castellana.

En cuanto a los datos del imperfecto de subjuntivo en las apódosis de las condicionales, este solo surge cuando el tiempo es compuesto, contexto en el que la solución conservadora es más resistente, como muestran los ejemplos $(12-14)$.

(12) I2: Allí el trabajo, buenísimo. Tenía [PS] tres habitacioncillas quizá más chicas que estas, un pasillo y un cuarto de aseo. No tenía más trabajo que ese, recoger, porque como to eran oficinas [HS:E [Asent]] pues no había suciedad ninguna, más que recoger los papeles y, y pasar la mopa que decían ahí, y ya está.E1: O_sea que vivía una vida regalada. [HS:I2 Una vida... Sí.] Menos que aquí.I2: Yo hubiera firmao pa to la vida, [HS:E1 ¿Sí?] ese trabajo. (Bacares).

(13) El: Y cuando dijo su hijo [Rndo: que no quería seguir estudiando] no....I1: [OTRAS-EM] [RISA] pa mí fue..., ipa mi si me [Rndo: hubieran dao] una paliza grande, grande no me hubiera sentao tan mal como me sentó aquella vez ! La verdad es que sí, jsí! (Aguilar de la Frontera).

(14) I1: Que, claro, había por_ejemplo | bueno, otros sí, otros no moría la madre, pero le venía hemorragia también, porque ya sabes que hay, que hay fetos que nacen de nalgas, como se dice. $\mathrm{Y}$ eso era mu difícil, a lo mejor cuando salía, salía el niño asfixiao. Y el crío ya. Niños morían muchos, muchos. Y ahora pos es mu difícil, [A-Inn] pues lo tenía así, que hace, todavía no hace aquí año y medio que [A-Inn]. Pues que se fue a la clínica, le hicieron sus cesáreas y el crío pues también. Y ella, pues entonces pues no. Es que las mujeres la mayoría | porque la, la mía mayor, porque mi mujer se pasó ya de | y hubo que llevarla a Jaén y en el hospital a el final ya | vaya, si no hubiera sío porque ya por la técnica de allí, pues hubiera nacío muerta, porque ya se había, se había desconectao de, de la madre. Y nació muy, con muy poco peso. Pero la otra no, la otra todavía estaba bien y fue la partera. (Arjona).

Los ejemplos $(12-14)$ ilustran esa permeabilidad del tiempo compuesto para mantener la forma - $r a$ en lugar del condicional, como ya alegaba Veiga (2006).

Si contemplamos otras herramientas dialectales, pero de la zona catalana y portuguesa, hallamos la siguiente casuística. Los textos catalanes del COD proveen datos de $-r a$ como sinónimo de $-s e$, aunque es cierto que presentan muy pocas ocurrencias. A continuación, exponemos algunos casos (15-21).

(15) Més de principis de segle io diria que després de, de la reforma que va fer Óscar Esplà, después de, después de la guerra. I se van restaurar Fundamentalment pa... pa ensaiar 
es cantors. I: també pa... pa que servira as polítics i as turistes, pa que vegueren la festa més tranquil·lament. (Elche).

(16) I, bueno, la diferència ademés de, de totes estes que t'ha dit e: $\mathrm{m}$ : és que no se corona la Mare Déu. Se fa un simulacre, com si se la coronara. (Elche).

(17) Bueno, Sueca és el poble on io visc des de que va nàixer. Ha eixit molt poquet d'ell:, aunque, encara que m'havera agradat eixir més. (Sueca).

(18) Soles té entre lo que és la, el mar i:, lo lo que és la mar i lo que és el poble n'hi ha, com si hi haveren dixat caure una roca allí $1 \mathrm{mig}$, pero que, que, ia, ia te dic, igual té vint metrosd'altura, no té res, i allí se va a passar el dia. (Sueca).

(19) I antes, ara això s'ha modernitzat una mica i encara és molt artesano. Se talla amb unes estisores com si siguera una bandereta però molt molt fineta molt fineta. (Morella).

(20) Va n'hi haver una pesta de les famoses a Morella i se va pensar de pujar la Mare de Déu en romeria a veure si aixina aconseguien que passara la peste i va passar, diuen que un milacre. (Morella).

(21) Molt-, hi ha gent que aguanta, però... Perqué si io sabera renegar i protestar i tot això, pues renegaria, protestaria, parlaria, pero no és una cosa que me ve, me ve... costa amunt, no? (Llíria).

Los ejemplos $(15$ - 21) muestran ocurrencias de la forma - ra como sustituto de -se, pero, al igual que en el ALPI, las únicas zonas catalanoparlantes que exhiben dicho fenómeno son las valencianas, desde Castellón, pasando por Valencia y llegando a Alicante. No encontramos muestras de este alomorfismo en Cataluña ni Baleares.

En el caso del portugués, el CORDIAL-SIN no ha dado ejemplos del alomorfo - ra, pero sí podemos constatar la vigencia del imperfecto de indicativo para contextos de IND 2. De todas las encuestas realizadas, por unanimidad, es el imperfecto de indicativo el que surge, como vemos en las siguientes ocurrencias.

(22) A gente, se o vento fosse contra, a gente tinha-se que ficar de fora. (Câmara de Lobos).

(23) E, por acaso, se o rei se quisesse saber bem a verdade, tinha os sete... Porque tinha vestido sete vestidos à sua filha, e todos os sete cada um de sua cor (Bandeiras).

(24) Porque a terra crua, a semente ficava ali em cima, vinha a bichareza, comia. E se ficasse com pouca terra em cima, a raiz ficava na terra que estava crua - ouviu? (Serpa). 
(25) Se tivesse, ele levava (Castro Laboreiro).

(26) Não podia ser muito menos porque senão, se fosse mais pequena, mais voltas dava (Moita de Martinho).

(27) "Ó senhor Halpagão, dava-me licença que fizesse aqui uma procura ao senhor engenheiro"? (Montalvo).

Las ocurrencias reproducidas en $(22-27)$ dan cuenta de que el portugués, a día de hoy, sigue prefiriendo el imperfecto de indicativo en lugar del condicional, cuyo uso se restringe a IND 2.

\section{ANÁLISIS}

Los mapas que hemos expuesto en el apartado anterior sugieren distintos comportamientos que merecen un análisis más profundo. En primer lugar, la desinencia en - ra se ha impuesto sobre -se en, prácticamente, todo el castellano. En segundo lugar, el condicional se expande por zonas del norte peninsular, aunque con una incidencia irregular según el contexto sintáctico en el que se inserte el verbo. Asimismo, los verbos modales modifican la distribución dialectal de la flexión verbal, ya que, por ejemplo, el portugués renuncia a $-s e$ favoreciendo el imperfecto de indicativo. De hecho, es este tiempo verbal el que surge en Baleares y el Rosellón en algunas subordinadas condicionales.

\section{1 -ra versus - se}

Una de las características que muestran los mapas sin lugar a dudas es la imposición de la innovación $-r a$ sobre -se en castellano, hasta tal punto que, independientemente de la formulación de la pregunta del ALPI o de otros atlas lingüísticos, que claramente podía favorecer una opción sobre la otra, los informantes se han comportado coherentemente, desechando la solución conservadora en prácticamente todo el territorio y en casi cualquier contexto sintáctico. No es el caso del catalán (excepto en Valencia) ni del portugués (pero sí de gran parte de Galicia). Es en el área gallego-portuguesa donde $-r a$ aún contiene la significación pluscuamperfecta.

Este desplazamiento de -se a favor de - $r a$ es un proceso lento que se inicia alrededor del siglo XIII y que, a tenor de los datos del ALPI, está completamente asentado en el habla oral y vernácula de mediados del siglo pasado. Es más, Rojo (1996) y 
Rosemeyer y Schwenter (2019) insinúan que, aunque el estándar acepte tanto -ra como - se para formar el imperfecto de subjuntivo, parece haber predilección por la forma conservadora en el lenguaje escrito frente al empleo de - $r a$ en el plano oral y más informal. Lo interesante es dilucidar cómo ha llegado una desinencia propia del pluscuamperfecto de indicativo a establecerse como imperfecto de subjuntivo. Si aplicamos los mapas del ALPI a la clasificación de SUBJ0 y SUBJ2, vemos que todas las preguntas se corresponden con un SUBJ0, excepto la del verbo modal, que indica una típica lectura de SUBJ2. En este sentido, todas las preguntas (excepto la del verbo modal, Yo quisiera ser rico) son las primeras en favorecer la expansión de la innovación $-r a$ a expensas de $-s e$. Es más, salvo la desiderativa Ojalá lloviese, las demás expresan una condicional que, como ya se ha dicho, es el primer contexto en promover la implantación de -ra sobre-se. Sin embargo, a pesar de la homogeneidad de los enunciados previstos en el ALPI, se advierten ciertas disonancias geográficas. En primer lugar, son las oraciones condicionales las que han promovido - $r a$ incluso en gallego y en el catalán de Valencia, que desecha -se en contraposición a Cataluña y el noreste de Aragón (así como Portugal) y, de manera esporádica, en zonas del centro-este peninsular. La distribución se ve modificada en las dos preguntas que no coinciden con la lectura condicional. En primer lugar, la frase desiderativa provoca la recuperación de -se en un área no desdeñable de Galicia, mientras que se mantiene férrea la alternativa en -ra en el español y el catalán de Valencia. Igualmente, la estrategia en -se es inamovible del portugués y del catalán hablado en Cataluña. En segundo lugar, la oración con el verbo modal reconfigura por completo las estrategias a nivel peninsular, ya que el condicional se asienta en toda el área catalanoparlante y el imperfecto de indicativo se impone en portugués y en parte del español occidental, así como de manera puntual en el resto de la Península Ibérica.

El uso de - $r a$ en esta frase está asociado a los valores iniciales de dicha desinencia, es decir, al IND2. Mientras que se ha conservado como modestia para los verbos modales, resulta arcaico en otras construcciones con otros verbos en el español peninsular (Veiga 2006). Es en América Latina donde aún se atestigua de manera más prolífica (De Sterck 2000, Rojo y Veiga 1999). Resalta la carencia total del alomorfo -se para esta lectura de IND2 en ninguna de las lenguas romances peninsulares. En su lugar, se ha sustituido por el condicional o bien por el imperfecto de indicativo.

El empleo del imperfecto de indicativo para lecturas condicionales como las de IND2, muy arraigadas en portugués y en el español coloquial, responde a otra reorganización temporal. Primero, Moreno de Alba (2006) aduce que el imperfecto de indicativo como sinónimo de condicional aparece muy esporádicamente a partir del siglo XII para valores modales y, con el tiempo, se irá extendiendo y haciendo general. 
Segundo, la irrupción de las formas de futuro a partir de la perífrasis infinitivo más HABEO y su homólogo en pasado infinitivo más HABEBAM supuso un reajuste temporal, ya que se introdujo una nueva forma de futuro y se inventó un tiempo nuevo (el condicional), que sirvió para hipótesis o como pasado del futuro.

La extensión del futuro de indicativo a lo largo de la Península Ibérica está documentada por Company (2006), Castillo (2000) o Saralegui (1983). De acuerdo con estas autoras, asistimos a un continuum geográfico, en el cual el catalán fue el primero en adoptar y generalizar el futuro. Seguidamente, el castellano hizo lo propio, mientras que el portugués aún conserva un estadio más conservador, ya que permite todavía la mesoclisis y no recurre con tanta frecuencia a dicho tiempo para expresar valores de posterioridad. En su lugar, el portugués opta por recurrir al futuro de subjuntivo o al presente de indicativo, según imponga la sintaxis. La permanencia del presente de indicativo en lugar del futuro parece tener su versión homóloga para un pretérito, ya que el condicional es sustituido por el imperfecto de indicativo, aunque no en cualquier contexto sintáctico. Es precisamente en lecturas de IND2 donde este tiempo desplaza al condicional. El español occidental de hace cien años exhibe el mismo patrón y, en cierta medida, concuerda con sus usos en futuros, ya que, como advierte Lara (2016), cuanto más al oeste del español peninsular nos hallemos, mayor será la probabilidad de encontrar tiempos de presente de indicativo en sustitución del futuro.

\subsection{Condicional por imperfecto de subjuntivo}

Sin duda, una de las características que posee el español peninsular septentrional es el desplazamiento del imperfecto de subjuntivo por el condicional. No obstante, si echamos un vistazo a las ilustraciones, el empleo del condicional crece o decrece según la frase que establece el cuestionario. La oración con la modalidad SUBJ 2 no da ocurrencias de condicional, es decir, no ha habido datos de Ojalá llovería, a excepción de un par de enclaves en la zona castellana septentrional. Es, no obstante, en las prótasis de las frases condicionales y en la única que exhibe una subordinada completiva donde el uso del condicional por el imperfecto se extiende por Navarra, La Rioja y el área norte de Castilla, casi con los mismos resultados. Sin embargo, la investigación de Pato (2003) revela que la implantación del condicional por el imperfecto de subjuntivo no surge en las oraciones condicionales, en contra de lo que se ha venido afirmando hasta ahora. En su estudio diacrónico y dialectal, el autor muestra que dicho tiempo verbal se inserta primero en las oraciones completivas, seguidas por las condicionales y, en última instancia, por las relativas con antecedente, las modales y las temporales. Asimismo, la ubicación geográfica más productiva la encuentra en 
el norte de Castilla, y no en la zona navarra o en contacto con el euskera, como se había advertido anteriormente.

Si echamos un vistazo a los resultados que se desprenden del Corpus Rural y Sonoro del Español Rural (COSER), cuyos datos se plasman en la obra de Pato (2003), podremos comparar la situación actual con las descripciones que hemos proporcionado del ALPI y los atlas posteriores. El mapa 10 que reproducimos a continuación está extraído de Pato (2003) y hace referencia a los lugares encuestados de los que el autor sacó los ejemplos.

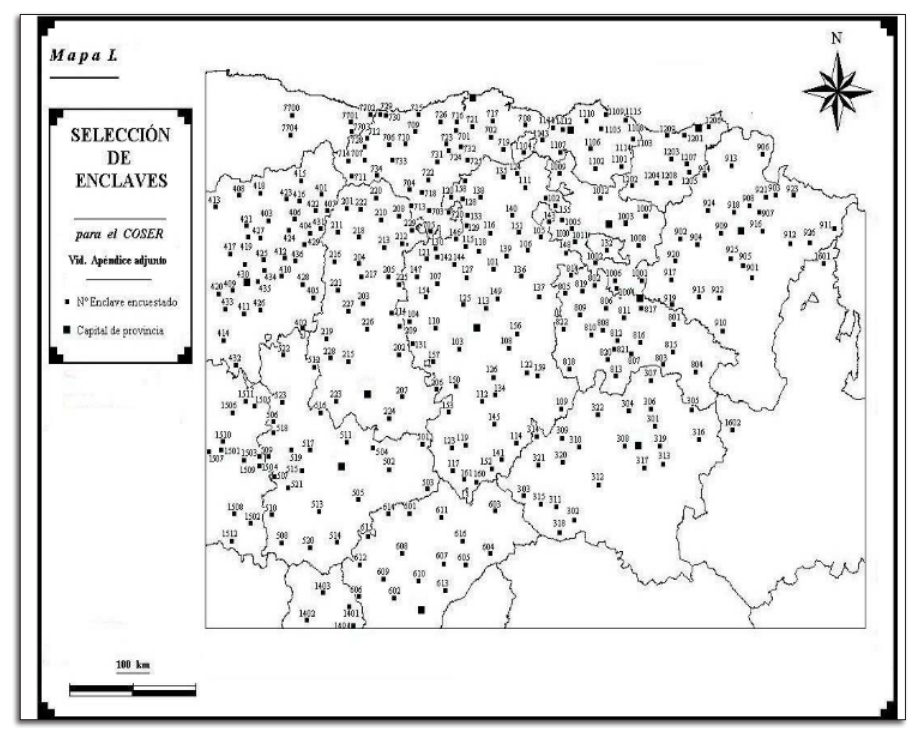

Mapa 10

Como se advierte, la sustitución del imperfecto de subjuntivo a favor del condicional se constriñe a zonas septentrionales, en concreto, Navarra, La Rioja, Cantabria, País Vasco y toda Castilla y León, salvo áreas occidentales. Según las investigaciones de Pato (2003), el empleo del condicional a costa de imperfecto de subjuntivo en la actualidad se circunscribe a lo indicado en el mapa 11 de a continuación, sacado precisamente de su tesis. 


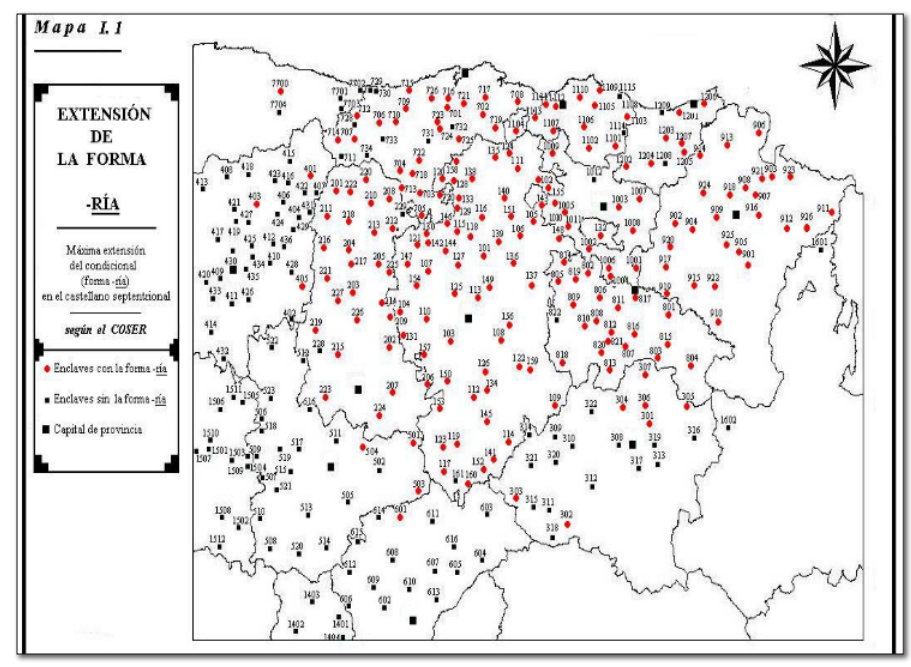

Mapa 11

El mapa 11 muestra que los puntos rojos son aquellos que exhiben el fenómeno de sustitución del imperfecto de subjuntivo favoreciendo el condicional, aunque, como ya hemos indicado, la incidencia se ve afectada por el tipo de oración subordinada en la que se inserte el tiempo verbal.

Una de las características de la aparición de este tiempo a costa del imperfecto de subjuntivo es el hecho de que no se circunscribe al español peninsular dialectal, sino que se da con mayor o menor incidencia en variedades latinoamericanas, con una valoración sociolingüística distinta de la de España. Tal y como aseguran Kany (1968) o Lipski (2007), el empleo del condicional en contextos donde el estándar impone el imperfecto de subjuntivo se atestigua sobre todo en zona andina y resulta un recurso lingüístico de todas las clases sociales, por lo que su valoración no posee la misma estigmatización que en el ámbito peninsular. Asimismo, los contextos sintácticos donde se asienta se corresponden con prótasis condicionales y no emerge tanto o nada en absoluto en completivas y otras subordinadas donde sí se esparce para el caso del norte peninsular. Este hecho, sin duda alguna, fomenta la hipótesis de la simetría a la que aludíamos, pero la explicación que se ha aducido para el español de América tiene que ver con las lenguas con las que mantiene contacto en los países donde este fenómeno se documenta. Ya que este uso tan solo se atestigua en los Andes y el quechua o el aimara exhiben el condicional en las prótasis, la explicación sobre su influencia es prácticamente automática. Sin embargo, De Granda (1998) no coincide al cien por cien en esta hipótesis y defiende que el uso del condicional por el imperfecto de subjuntivo 
es el resultado del contacto lingüístico con las lenguas indígenas, sumado al hecho de que los propios colonizadores ya traían dicho fenómeno de la Península Ibérica. A nuestro modo de ver, existen dos problemas fundamentales en la crítica de De Granda. La primera es que, si los colonizadores hubiesen exportado dicho fenómeno, lo más probable es que lo halláramos en todo el español americano y no solo en el Altiplano. La segunda es que, si los colonizadores hubiesen exportado dicho fenómeno, esperaríamos su aparición en otros contextos sintácticos, como completivas, finales, etcétera, ya que, como bien aducía Pato (2003), el contexto más probable para dicha sustitución era la subordinación sustantiva y no la condicional. Recordemos que, en el caso del español americano, tan solo se da en las condicionales, a imitación de las lenguas indígenas de su entorno, donde el tiempo condicional surge en las prótasis, es decir, exactamente en los mismos contextos sintácticos.

Es más, la sustitución de una forma subjuntiva por un condicional en las prótasis de las condicionales está más o menos extendida y no se circunscribe solo al español. En el caso del francés, Tesnière (1959) asegura que se da dialectalmente en la variedad europea; en el italiano, son Vincent \& Bentley (1995) o Rohlfs (1968) quienes apuntan al mismo hecho. Pero las lenguas romances no son exclusivas a este respecto, sino que el mismo inglés, por ejemplo, también presenta la misma casuística dialectal e incluso en fases anteriores de su historia aparece el auxiliar would en prótasis de textos escritos y literarios, sin que contraviniera el estándar (Lass 1999).

\subsection{Indicativo por subjuntivo}

Otro de los fenómenos que arrojan los resultados del ALPI es el declive del modo subjuntivo por el indicativo en Baleares y en la zona francesa de habla catalana. El declive en el área en contacto con el francés puede atribuirse a esta lengua, ya que también ha desplazado el modo subjuntivo favoreciendo el indicativo en casi todos los contextos, siendo el presente el que más se resiste (Le Goffic 1997). Sin embargo, el pretérito imperfecto de subjuntivo se constriñe a la lengua muy culta y es la forma de indicativo la que se ha impuesto en cualquier contexto. No obstante, los datos de Baleares nos indican que el declive del imperfecto de subjuntivo por el de indicativo se engloba en una tendencia universal en la cual el subjuntivo va retrocediendo y repartiendo sus usos a los tiempos correspondientes del indicativo. De acuerdo con Greenberg (2005), la desaparición de un elemento lingüístico dado (como una marca de persona, tiempo y modo verbales, etc.) suele responder a una jerarquía, en la cual el plural es más proclive al sincretismo o la analogía frente al singular; la desinencia de tercera persona suele ir copando las demás, siendo la primera persona del singular 
la última en ceder; el presente tiende a adoptar usos de pasado y futuro; o el modo indicativo termina haciendo referencia también a lecturas irrealis que suelen caracterizar el subjuntivo o el imperativo.

Si nos atenemos a la situación de otros idiomas, observamos que el subjuntivo ha decrecido a lo largo del tiempo, ha desaparecido o se mantiene de manera anecdótica en construcciones muy específicas. Lombardi Vallauri (2003) o Le Goffic (1997) apuntan a una progresión cada vez más acentuada en la desaparición del subjuntivo en francés e italiano. En el caso del primero, son los tiempos de pasado los que más se ven afectados, mientras que en el caso del segundo son los presentes los que más sucumben. Sin lugar a dudas, es el inglés el que mejor ejemplifica la desaparición del subjuntivo a favor de otras estrategias o del indicativo. Es tan solo con el verbo to be ('ser / estar') en la 1sg y 3sg donde se mantiene una forma de imperfecto de subjuntivo en oraciones condicionales.

El desplazamiento del subjuntivo por el indicativo no solo se concentra en dicha oposición sintáctica, sino que alcanza la dicotomía semántica realis - irrealis que, en principio, fundamenta la clasificación sintáctica de indicativo y subjuntivo (si bien existen muchas contradicciones entre sintaxis y semántica a este respecto). El mismo condicional puede ser sustituido en ocasiones por el imperfecto de indicativo $\mathrm{y}$, aunque el condicional se inserte sintácticamente en el modo indicativo, en realidad alude a un modo irrealis. En consecuencia, es el modo irrealis (independientemente del modo sintáctico) el que se ve desfavorecido por el realis. Este hecho se documenta en nuestros resultados sobre todo en el portugués, como observamos en $(22-27)$.

Pero la pérdida paulatina del subjuntivo puede incluso verse en sus tiempos verbales. El español de América exhibe de manera generalizada la discordancia temporal entre el verbo de la principal y de la subordinada cuando esta última posee un tiempo flexionado en modo subjuntivo (Kany 1969, Lipski 2007). El tiempo que se pierde es el de imperfecto de subjuntivo a favor del empleo del presente de subjuntivo, como se ven en $(28-29)$.

(28) Me dijo que venga a casa.

(29) El año pasado mi jefe me ordenó que vaya a una reunión en Italia y tuve que ir.

Los ejemplos (28 - 29) muestran que, aunque el subjuntivo no se ha perdido o no tiene visos de retroceder ante el indicativo, como sí ocurre en italiano y francés, sus tiempos verbales sí que decrecen en número, ya que el pasado apenas surge en dichas variedades americanas a pesar de que la consecutio temporum obliga a emplearlo. 


\subsection{Verbos modales}

El mayor cambio en la elección de la forma verbal lo provoca la única pregunta que prevé un lexema modal, como es querer. En este caso, el área portuguesa ha optado casi unánimemente por un imperfecto de indicativo, opción que también se inserta en el castellano occidental y, de manera esporádica, en el noreste de Aragón y el sureste de Andalucía. La respuesta coincidente con un imperfecto de subjuntivo es la mayoritaria del español, el gallego, la zona asturiana y no se documenta en zona catalana, ni siquiera en aquella región donde sí se empieza a recurrir al morfema - $r a$ en otros contextos. De acuerdo con Veiga (2006) o De Sterck (2000), la posibilidad de usar la desinencia de imperfecto de subjuntivo en contextos sintácticos en los que, a priori, solo es posible el condicional solo se documenta en verbos modales, como deber, poder o querer. Esta opción no sería más que un vestigio de una situación anterior a la que conocemos actualmente, ya que aún se mantiene en la oración principal una forma totalmente vigente para las condicionales o las desiderativas en época medieval. No es el caso del español de América, donde, de acuerdo con Lipski (2007) o Kany (1968), la forma en - ra puede seguir apareciendo en cualquier verbo de la apódosis como reliquia sintáctica. La diferencia por tanto entre ambas variedades es la ampliación en su uso: mientras que el español peninsular tan solo lo permite en los modales, el español americano es capaz de universalizarlo a cualquier verbo.

La conservación de un estadio anterior de la lengua precisamente en verbos modales no parece tampoco arbitrario, ya que esta clase de verbos suele presentar comportamientos propios que los diferencian del resto de lexemas, salvo si estos funcionan como auxiliares. Esta tendencia es universal y, de hecho, dos de los idiomas que mejor ejemplifican el carácter propio de los verbos modales son el inglés y el alemán. Ambas lenguas poseen características comunes en la configuración sintáctica de sus verbos modales. En primer lugar, el verbo modal favorece en su expresión pretérita el uso del pasado simple en contra del empleo no marcado del perfecto en alemán. En inglés también se promociona la utilización del pretérito incluso en contextos donde se optaría por el perfecto compuesto en otros verbos. En segundo lugar, las oraciones subordinadas que se predican de verbos modales que aparecen en la principal anulan el surgimiento de la preposición to (en inglés) / $z u$ (en alemán) que suele anteceder al verbo de la subordinada o incluso su flexión en -ing en el caso del inglés. Además, los verbos modales pueden incluso ser motores del cambio lingüístico, ya que el rumano, el inglés y el griego, sin ir más lejos, comparten la gramaticalización de sus correspondientes verbos con significación querer como auxiliares de una expresión de futuro. 
En cualquier caso, los verbos modales son más reticentes a las innovaciones; lo vemos en el mantenimiento del pretérito por el perfecto cuando la tendencia es la contraria o el no surgimiento de la preposición en la subordinada cuando la norma es la opuesta. En el español, los verbos modales, al menos en el siglo xx, aún pueden desarrollar una morfología de imperfecto de indicativo en una oración principal, como en época medieval. Aunque pueden ser sustituidos por formas de condicional, estas conviven con el uso anterior.

\section{CONCLUSIONES}

Los datos nos han mostrado que la distribución dialectal en las lenguas romances de la Península Ibérica a lo largo del siglo xx en lo que respecta al imperfecto de subjuntivo puede materializarse de distintas formas. La solución etimológica en -se se mantiene férrea en catalán y portugués, mientras que la innovación en - $r a$ es hegemónica en castellano, mayoritaria en gallego e incluso en el catalán de la Comunidad Valenciana. Sin embargo, Baleares y el catalán septentrional recurren al imperfecto de indicativo en ciertos contextos sintácticos, al igual que el portugués cuando se trata de un IND 2. El caso del catalán del Rosellón, no obstante, obedece a la tendencia tipológica que establece que el indicativo va acaparando usos del subjuntivo gradualmente. En cuanto al español, el norte exhibe formas de condicional por imperfecto de subjuntivo, aunque con una incidencia irregular, según el contexto sintáctico. Sin embargo, su comportamiento sintáctico no es el mismo que el del español andino, ya que el condicional es más probable en una completiva antes que en una condicional, lo cual no es el caso del español de los Andes, donde el condicional tan solo se documenta en las prótasis por influencia de las lenguas indígenas con las que el español convive en dicha área. Lo relevante es la imposición de - $r a$ sobre -se en prácticamente todo el español y su permanencia como IND 2 en verbos modales, como vestigio del comportamiento gramatical medieval. Aunque hemos observado usos de $-r a$ como IND 2 con otros verbos en el ALEA y en el ALPI, las ocurrencias son minoritarias y, desde luego, no imitan la sistematicidad del español de América en este sentido. El único contexto donde el imperfecto de subjuntivo acabado en - $r a$ sigue vigente sin connotar arcaísmo y con la capacidad de intercambiarse con el condicional es cuando el tiempo es perfecto.

\section{BIBLIOGRAFÍA}

ALCyL = Alvar, M. (1999): Atlas lingüistico de Castilla y León. Salamanca: Junta de Castilla y León. 
ALEA = Alvar, M. et al. (1961-1973): Atlas lingüístico y etnográfico de Andalucía . Granada: Universidad de Granada / CSIC.

ALEANR = Alvar, M. (1979-1983): Atlas lingüístico y etnográfico de Aragón, Navarra y Rioja. Zaragoza / Madrid: Diputación Provincial de Zaragoza / CSIC.

ALECANT = Alvar, M. (1995): Atlas lingüistico y etnográfico de Cantabria . Madrid: Arco Libros.

ALEC-MAN = García Mouton, P. y F. Moreno Fernández (1988 - 1994): Atlas lingüístico (y etnográfico) de Castilla-La Mancha. Alcalá de Henares: Universidad de Alcalá.

ALPI = Heap, D. (2003): Atlas lingüistico de la Península Ibérica. Ontario: University of Western Ontario.

Badia i Margarit, A. (1994): Gramàtica de la llengua catalana. Barcelona: Enciclopèdia Catalana.

CANO, R. (1990): "Sobre la historia del subjuntivo en español", in M. A. A. Martínez (ed.): Actas del Congreso de la Sociedad Española de Lingüística. Madrid: Gredos, pp. 339-353.

Castillo, M. (2000): "Distribución de las formas analíticas y sintéticas de futuro y condicional en español medieval”, in M. T. Echenique y J. Sánchez Méndez (eds.): Actas del V Congreso Internacional de Historia de la Lengua Española. Madrid: Gredos, pp. 543-549.

COD = Viaplana, J. et al. (2007): COD. Corpus Oral Dialectal. Barcelona: Promociones y Publicaciones Universitarias.

Company, C. (2006): "Los futuros y condicionales", in C. Company (dir.): Sintaxis histórica de la lengua española. México DF: UNAM / Colmex, pp. 349-418.

CORDIAL-SIN = Martins, A. M. (coord.) (2010): CORDIAL-SIN: Corpus Dialectal para o Estudo da Sintaxe / Syntax-oriented Corpus of Portuguese Dialects. Lisboa: Centro de Linguística da Universidade de Lisboa.

COSER = Fernández-Ordóñez, I. (dir.) (2005-): Corpus Oral y Sonoro del Español Rural. Madrid: Universidad Autónoma de Madrid. www.corpusrural.es.

De Granda, G. (1998): “Condicionamientos internos y externos de un proceso de variación morfosintáctica en el español andino. Potencial / subjuntivo en estructuras condicionales", Boletín de Filología 37 (1), pp. 547-564.

De StercK, G. (2000): Registros y áreas geográficas en lingüística. Valores y usos de las formas verbales en -ra, -se, -ría y-re. Salamanca: Universidad de Salamanca. 
GreenberG, J. (2005): Language universals. Nueva York / Berlín: Mouton de Gruyter. https://doi.org/10.1515/9783110899771.

HARRIS, M. B. (1971): "The history of the conditional complex from Latin to Spanish: some structural considerations", Archivum Linguisticum 2, pp. 25-33.

Kany, C. E. (1969): Sintaxis hispanoamericana. Madrid: Gredos.

LARA, V. (2016): "La expresión del futuro en las lenguas romances de la Península Ibérica”, Boletín de la Real Academia Española XCVI (CCCXIV), pp. 529-558.

LASs, R. (1999): The Cambridge history of the English language. Cambridge: Cambridge University Press.

LE GofFic, P. (1997): Les formes conjuguées du verbe en français oral et écrit. París: Ophrys.

LIPSKI, J. (2007): El español de América. Madrid: Cátedra.

LOMBARDI VALlAURI, E. (2003): "Vitalità del congiuntivo nell'italiano parlato", in N. Maraschio y T. Poggi (eds.): Atti del XXXIV Congresso Internazionale di Studi della Società di Linguistica Italiana. Roma: Bulzoni, pp. 609-634.

LuQuet, G. (1988): Systématique historique du mode subjonctif espagnol. París: Klincksieck.

Montero, E. (1989): Gonzalo de Berceo y el Libro de Alexandre. Aproximación al sistema verbal de la época desde los esquemas condicionales. Santiago de Compostela: Universidade de Santiago de Compostela.

Moreno de AlbA, J. G. (2006): "Valores de los tiempos pasados de indicativo y su evolución”, in C. Company (dir.): Sintaxis de la lengua española. México: UNAM / Colmex, pp. 5-92.

NARbona, A. (1990): Las subordinadas adverbiales impropias del español. Causales y finales, comparativas y consecutivas, condicionales y concesivas. Málaga: Ágora.

PATO, E. (2003): La sustitución de cantara/cantase por cantaría y cantaba en el castellano septentrional peninsular. Madrid: Universidad Autónoma de Madrid.

RIDRUEJo, E. (1979): “La forma verbal en -ra en español del siglo XIII", Cuadernos de investigación filológica 5, pp. 23-38.

RoHLFs, G. (1968): Grammatica storica della lingua italiana e dei suoi dialetti. Turín: Einaudi.

Rojo, G. y A. VeIGA (1999): "El tiempo verbal. Los tiempos simples”, in I. Bosque y V. Demonte (eds.): Gramática descriptiva de la lengua española. Madrid: Espasa, pp. 2867-2934.

RoJo, G. (1974): “La temporalidad verbal en español”, Verba 1, pp. 63-149. 
Rojo, G. (1996): "Sobre la distribución de las formas llegara y llegase en español actual", in M. Casado et al. (eds.): Scripta philologica in memoriam Manuel Taboada Cid. La Coruña: Universidade da Coruña, pp. 677-691.

RoSEMEYeR, M. y S. SCHWENTER (2019) : "Entrechment and persistence in language change: the Spanish past subjunctive", Corpus Linguistics and Linguistic Theory 15 (1), pp. 167-204.

SARALEGUi, C. (1983): "Morfología del futuro y condicional castellanos: poliformismo antiguo y fijación lingüística", Medioevo Romanzo 8 (3), pp. 419-459.

SolÀ, J. (2002): Gramàtica del català contemporani. Barcelona: Empúries.

TeSniÈre, L. (1959): Éléments de syntaxe structurale. París: Klincksieck.

Veiga, A. (2006): "Las formas verbales subjuntivas. Su reorganización modo-temporal", in C. Company (dir.): Sintaxis histórica de la lengua española. México DF: UNAM / Colmex, pp. 95-240.

VinCENT, N. y D. BentLey (1995): "Conditional and subjunctive in Italian and Sicilian: a case study in the province of Palermo", in A. Giacalone y G. Crocco (eds.): From pragmatics to syntax. Modality in second language acquisition. Tübingen: Gunter Narr, pp. 11-33.

WheELER, M. W. ET AL. (1999): Catalan: a comprehensive grammar. Londres: Routledge. https://doi.org/10.4324/9780203300275.

Wright, L. O. (1933): "The earliest shift of the Spanish -ra verb form from the indicative function to the subjunctive: 1000-1300 a.d.", Language 9, pp. 265-268. https://doi.org/10.2307/409354. 